\title{
Fabrication and Manipulation of Gold 1D Chain Assemblies Using Magnetically Controllable Gold Nanoparticles
}

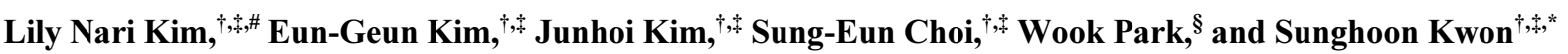 \\ ${ }^{\dagger}$ School of Electrical Engineering and Computer Science, Seoul National University, Seoul 151-744, Korea \\ *Inter-University Semiconductor Research Center (ISRC), South Korea. *E-mail: skwon@snu.ac.kr \\ ${ }^{\S}$ Department of Electronics and Radio Engineering, Institute for Laser Engineering, Kyung Hee University, \\ Yongin 446-701, Korea \\ ${ }^{\#}$ Department of Livestock Business and Marketing Economics, College of Animal Bioscience and Technology, \\ Konkuk University, Seoul 143-701, Korea \\ Received July 25, 2012, Accepted August 30, 2012
}

\begin{abstract}
We have developed magnetically controllable gold nanoparticles by synthesizing superparamagnetic $\mathrm{Fe}_{3} \mathrm{O}_{4}$ core/gold shell nanoparticles. The core/shell particles have the capability of forming gold 1D chains in the presence of an external magnetic field. Here we demonstrate dynamic and reversible self-assembly of the gold 1D chain structures in an aqueous solution without any templates or physical or chemical attachment. The spatial configuration of gold chains can be arbitrarily manipulated by controlling the direction of a magnetic field. This technique can provide arbitrary manipulation of gold $1 \mathrm{D}$ chains for fabrication purpose. To demonstrate this capability, we present a technique for immobilization of the gold particle chains on a glass substrate.
\end{abstract}

Key Words : Magnetic core/gold nanoshell, Gold nanoparticle, Self-assembly, Magnetic manipulation

\section{Introduction}

The ability to assemble noble metal nanoparticles is an important area of study due to their unique localized surface plasmon resonance (LSPR) properties ${ }^{1-3}$ and their applications in plamonics, ${ }^{4,5}$ photonics, ${ }^{6}$ surface-enhanced spectroscopies. ${ }^{7,8}$ Additionally, ordered assemblies of metal nanoparticles can provide distinct optical and electronic properties compared to single particles or disordered particle conglomeration. ${ }^{9,10}$ In particular, the organization of metal nanoparticles into 1D chain structures has important applications in areas such as LSPR coupling, plasmon-based waveguide, ${ }^{11,12}$ electron tunneling between neighboring particles. ${ }^{13}$ Owing to their unique characteristics, many attempts have been made to make gold $1 \mathrm{D}$ chain structures using various approaches. The conventional fabrication techniques for fabricating 1D particle chains utilize templates such as phospholipids, ${ }^{14,15}$ polymers, ${ }^{16}$ DNA,${ }^{17}$ peptide nanotubes, ${ }^{18}$ carbon nanotubes. ${ }^{19}$ However, these approaches require complicated multistep methods including preparation of functionalized templates and attachment of metal nanoparticles in order to assemble particles onto the templates. Also, the large-scale fabrication and the arbitrary control of chain direction are very difficult. 1D chains can be also generated by spontaneous self-assembly of nanoparticles which have magnetic ${ }^{20}$ or electric dipoles. ${ }^{21}$ The use of electric forces is a promising technique for the formation and manipulation of chain structures, but this technique has constraints, including the potential for inducing electrochemical process and localized heating. ${ }^{22}$ In comparison to other methods for generating gold particle chains, the magnetic assembly method is instantaneous and enables large-scale fabrication ${ }^{23}$ with a high degree of spatial control. ${ }^{24-26}$

Here we report the fabrication and arbitrary manipulation of gold particle chain assemblies using superparamagnetic core/gold shell nanoparticles in an aqueous solution. We overcome the limitations of conventional fabrication techniques by fabricating gold 1D chain structures using a magnetic field, which greatly simplifies the fabrication process. Using our method, millions of chains can be created simultaneously in a few seconds, in the absence of templates or any attachment process. We also demonstrate arbitrary manipulation of chain orientation by controlling the direction of a magnetic field. Moreover, we provide the technique for immobilization of chain structures on a glass substrate.

(a)

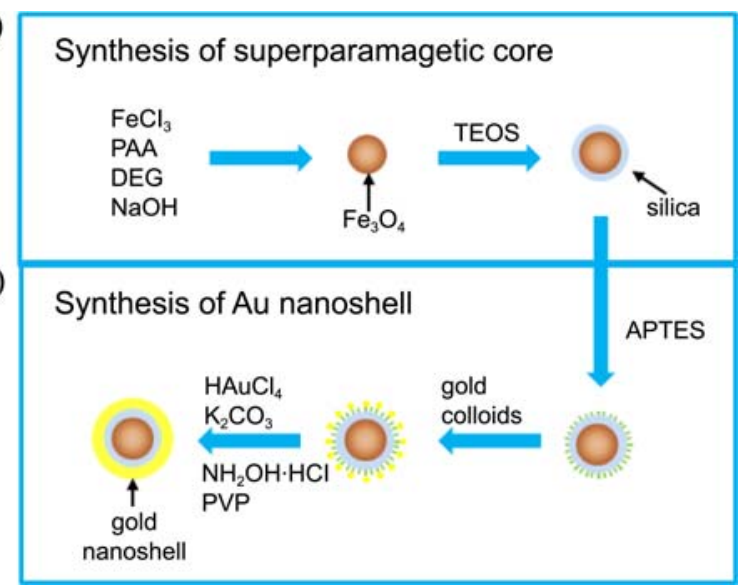

Figure 1. The synthesis of magnetically controllable gold nanoparticles. (a) A schematic diagram overviewing the synthesis of superparamagnetic $\mathrm{Fe}_{3} \mathrm{O}_{4}$ core and (b) gold shell layer. 


\section{Experimental}

The schematic diagram of synthesis of superparamagnetic core/gold shell is shown in Figure 1.

Materials. $\mathrm{FeCl}_{3}$ (anhydrous, 97\%, Fisher Scientific), diethylene glycol (DEG, 99\%, Daejung), poly(acrylic acid) (PAA, Mn = 1,800, Sigma-Aldrich), $\mathrm{NaOH}$ (pellet, 98\%, Daejung), tetraethyl orthosilicate (TEOS, 99.999\%, SigmaAldrich), $\mathrm{NH}_{4} \mathrm{OH}$ (28\%, Daejung), EtOH (99.9\%, Daejung), 3-aminopropyltriethoxysilane (APTES, 99\%, Sigma-Aldrich), $\mathrm{HAuCl}_{4} \cdot 3 \mathrm{H}_{2} \mathrm{O}(99.9 \%$, Sigma-Aldrich), tetrakis(hydroxymethyl)phosphonium chloride (THPC, $80 \%$ in $\mathrm{H}_{2} \mathrm{O}$, SigmaAldrich), $\mathrm{K}_{2} \mathrm{CO}_{3}, \mathrm{NH}_{2} \mathrm{OH} \cdot \mathrm{HCl}(99 \%$, Sigma-Aldrich), poly(vinyl pyrrolridone) (PVP, $\mathrm{Mn}=10,000$, Sigma-Aldrich)

Preparation of Stock Solutions. $\mathrm{A} \mathrm{FeCl}_{3} / \mathrm{DEG}$ stock solution is prepared by dissolving $20 \mathrm{mmol} \mathrm{FeCl}_{3}$ in $50 \mathrm{~mL}$ of DEG and heating it at $120^{\circ} \mathrm{C}$ for $1 \mathrm{~h}$ under a nitrogen atmosphere. A NaOH/DEG stock solution is also prepared by dissolving $50 \mathrm{mmol}$ of $\mathrm{NaOH}$ in $50 \mathrm{~mL}$ of DEG and heating it at $120^{\circ} \mathrm{C}$ for $1 \mathrm{~h}$ under a nitrogen atmosphere.

Synthesis of Superparamagnetic $\mathrm{Fe}_{3} \mathrm{O}_{4}$ Nanoparticles. The superparamagnetic $\mathrm{Fe}_{3} \mathrm{O}_{4}$ core nanoparticles with tunable size are synthesized by using a high-temperature hydrolysis reaction with PAA as a surfactant. ${ }^{27}$ In a typical synthesis, a mixture of $288 \mathrm{mg}$ of PAA, $1 \mathrm{~mL}$ of $\mathrm{FeCl}_{3}$ stock solution and $15 \mathrm{~mL}$ of DEG is heated to $220^{\circ} \mathrm{C}$ in a nitrogen atmosphere for $90 \mathrm{~min}$ with vigorous stirring to form a transparent, light yellow solution. Then, $1.8 \mathrm{~mL}$ of $\mathrm{NaOH} /$ DEG stock solution is injected into the above solution which slowly turned black after about $2 \mathrm{~min}$. The resulting mixture is further heated for $1 \mathrm{~h}$ to yield about $120 \mathrm{~nm} \mathrm{Fe}_{3} \mathrm{O}_{4}$ colloids. Introduction of $\mathrm{NaOH}$ into the hot mixture of DEG, $\mathrm{FeCl}_{3}$ and PAA produces water molecules and also increases the alkalinity of the reaction system and both results favor the hydrolysis of $\mathrm{FeCl}_{3}$. The average size of the superparamagnetic core particles can be precisely controlled simply by varying the amount of $\mathrm{NaOH}$. The synthesized $\mathrm{Fe}_{3} \mathrm{O}_{4}$ colloids are first washed with a mixture of deionized (DI) water and ethanol, then with pure water several times, and finally dispersed in $3 \mathrm{~mL}$ of DI water.

Silica Coating on the $\mathrm{Fe}_{3} \mathrm{O}_{4}$ Nanoparticles. The silica coating on $\mathrm{Fe}_{3} \mathrm{O}_{4}$ nanoparticles is performed using a modified Stöber method. ${ }^{28}$ Typically, $3 \mathrm{~mL}$ of a $\mathrm{Fe}_{3} \mathrm{O}_{4}$ colloids solution is mixed with ethyl alcohol $(20 \mathrm{~mL}), \mathrm{NH}_{4} \mathrm{OH}(28 \%$, $1 \mathrm{~mL})$. TEOS $(0.1 \mathrm{~mL})$ is injected into the solution. After 20 $\min$, TEOS $(0.1 \mathrm{~mL})$ is injected one more time. After the reaction finished, the $\mathrm{Fe}_{3} \mathrm{O}_{4} / \mathrm{SiO}_{2}$ particles are collected by magnetic separation, followed by rinsing with ethanol 3 times and finally dispersed in $3 \mathrm{~mL}$ of EtOH.

APTES-Functionalization of $\mathrm{Fe}_{3} \mathrm{O}_{4} /$ Silica Surface. APTES can be covalently bonded to the surface of silica. The $3 \mathrm{~mL}$ of $\mathrm{Fe}_{3} \mathrm{O}_{4} /$ silica colloid solution is added to $50 \mathrm{~mL} \mathrm{EtOH}$, placed in a polypropylene bottle and stirred rapidly. $0.5 \mathrm{~mL}$ of APTES is added to the suspension with continuous stirring for $5 \mathrm{~min}$. The mixture is boiled for $2 \mathrm{~h}$. Total evaporation of the EtOH during the APTES functionalization is prevented by the continuous addition of EtOH. Next, the functionalized magnetic core particles are washed 3 times in EtOH by magnetic separation. The functionalized particles are finally redispesed in $15 \mathrm{~mL}$ of EtOH.

Synthesis of THPC-Gold Solution. The synthesis of THPC-gold solution composed of 1-3 nm Au colloids is performed as follows: $2 \mathrm{~mL}$ of $1 \mathrm{M} \mathrm{NaOH}$ is added to 180 $\mathrm{mL}$ of DI water, followed by the addition of $4 \mathrm{~mL}$ THPC solution (prepared by adding $50 \mathrm{~L}$ of $80 \%$ THPC to $4 \mathrm{~mL} \mathrm{DI}$ water). The mixture is stirred for $10 \mathrm{~min}$ and then $8 \mathrm{~mL}$ of $25.4 \mathrm{mM} \mathrm{HAuCl}_{4}$ is added rapidly. The color of the mixture changed from yellow to dark brown within a few seconds, indicating the formation of THPC-gold nanoparticles.

Attachment of THPC-Gold Nanoparticles to an APTESFunctionalized Magnetic Core. In order to attach the THPCgold nanoparticles, $20 \mathrm{~mL}$ of a THPC-gold solution is ultrasonicated for $1 \mathrm{~min}$. During ultrasonication, $2 \mathrm{~mL}$ of $1 \mathrm{M}$ $\mathrm{NaCl}$ is added, followed by the addition of $0.6 \mathrm{~mL}$ of APTESfunctionalized magnetic core particles. After continuous ultrasonication for an additional $2 \mathrm{~min}$, the solution is allowed to reach equilibrium for $12 \mathrm{~h}$. The gold-decorated $\mathrm{Fe}_{3} \mathrm{O}_{4}$ nanoparticle solution is washed with EtOH 3 times to remove excess THPC-gold nanoparticles and then the particles are redispersed in $3 \mathrm{~mL}$ EtOH.

Preparation of Gold Plating Solution. Gold plating solution is prepared for the growth of gold nanoshells. First, $0.05 \mathrm{~g}$ of $\mathrm{K}_{2} \mathrm{CO}_{3}$ is dissolved in $200 \mathrm{~mL}$ DI water and then the solution is stirred for $10 \mathrm{~min}$. Next, $3 \mathrm{~mL}$ of $25.4 \mathrm{mM}$ $\mathrm{HAuCl}_{4}$ is added to the solution. The solution is aged for 24 $\mathrm{h}$ in the dark before it is used.

Gold Nanoshell Synthesis. The THPC-gold particles attached to the silica surface are used as nucleation sites for the further reduction of gold, resulting in deposition of a gold nanoshell layer on the magnetic core particle. ${ }^{3,29}$ The $0.3 \mathrm{~mL}$ of gold-decorated magnetic core nanoparticles are mixed with $3 \mathrm{~mL}$ of gold plating solution and then $1 \mathrm{~mL}$ of $1 \% \mathrm{PVP}$ is added. Next, $261 \mu \mathrm{L}$ of $\mathrm{NH}_{2} \mathrm{OH} \cdot \mathrm{HCl}$ is added to the mixture to reduce gold ions on gold colloids of a silica surface exclusively. The reduction is performed during $1 \mathrm{~h}$, allowing for complete consumption of $\mathrm{Au}^{3+}$. The magnetic core/gold nanoshell particles are washed with DI water 3 times by magnetic separation and finally redispersed in 3 $\mathrm{mL}$ of water.

Immobilization of Gold 1D Chains on a Glass Substrate. The amino-terminating APTES monolayer are self-assembled on a glass substrate by dipping the substrate in a 1\% APTES solution of EtOH for $1 \mathrm{~h}$, followed by rinsing in EtOH. Finally, the substrate is baked at $80^{\circ} \mathrm{C}$ for $1 \mathrm{~h}$ to complete the $\mathrm{Si}-\mathrm{O}$ bond formation. The magnetic core/gold nanoshell particles are assembled on the amine-treated glass substrate by applying a magnetic field for $1 \mathrm{~h}$. The substrate is thoroughly washed with DI water immediately after the magnetic field is removed.

\section{Results and Discussions}

Figure 2(a)-(c) shows a series of TEM images showing gold nanoshell growth on a $\mathrm{Fe}_{3} \mathrm{O}_{4} /$ silica nanoparticle with 

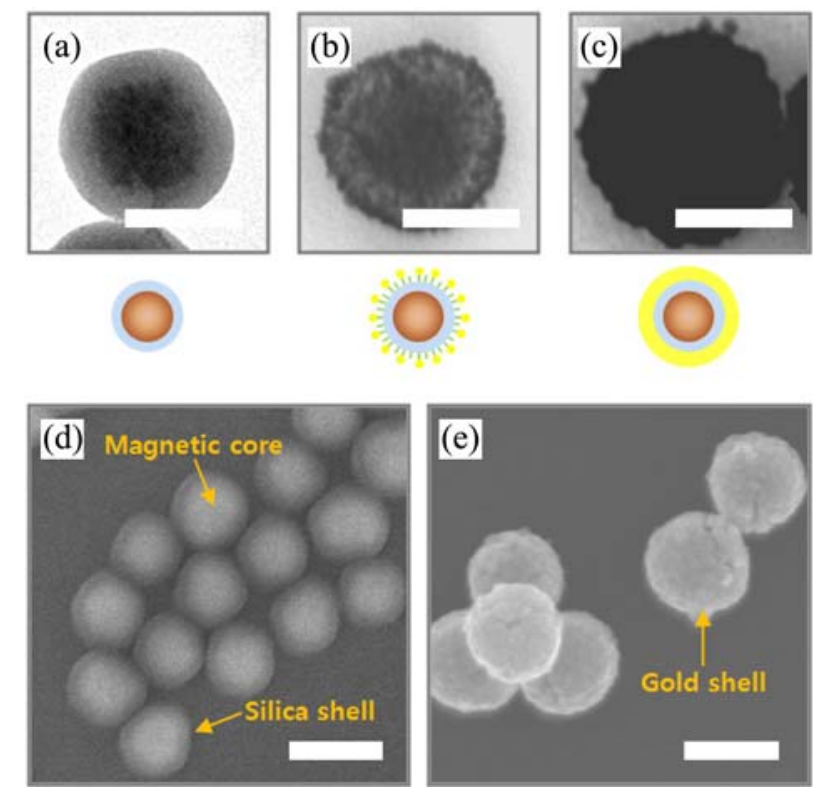

Figure 2. (a-c) A series of TEM images showing gold nanoshell growth on $\mathrm{Fe}_{3} \mathrm{O}_{4} /$ silica nanoparticle with different coverage. The scale bars indicate $100 \mathrm{~nm}$. (d) FE-SEM image of superparamagnetic $\mathrm{Fe}_{3} \mathrm{O}_{4}$ core/silica shell nanoparticles. (e) FE-SEM image of magnetic core/gold shell nanoparticles. The scale bars indicate 200 $\mathrm{nm}$ in figure 2(d) and 2(e).

different Au coverage. Initially, the seed gold colloid increase in size as reduction proceeds. Then the seed colloids begin to coalesce, until the complete formation of a continuous gold nanoshell on a silica surface can be observed. The complete nanoshell is shown in Figure 2(c). The average diameter of $\mathrm{Fe}_{3} \mathrm{O}_{4} /$ silica nanoparticles is $150 \pm 10 \mathrm{~nm}$ and the average thickness of gold nanoshells is approximately $25 \mathrm{~nm}$. The thickness of the metal nanoshells can be controlled by varying the volume ratio of a magnetic core particle solution and a gold plating solution, correlated with optical properties. ${ }^{30,31}$ Figure 2(d) and (e) shows FE-SEM images of superparamagnetic $\mathrm{Fe}_{3} \mathrm{O}_{4}$ core/silica shell nanoparticles and magnetic core/gold shell nanoparticles respectively.

The UV-vis spectra of the superparamagnetic $\mathrm{Fe}_{3} \mathrm{O}_{4}$ core (blue line) and $\mathrm{Fe}_{3} \mathrm{O}_{4}$ core/gold shell nanoparticles (red line) suspended in water are shown in Figure 3(a). The deposition of gold onto magnetic core leads to a shift in the SPR absorption. The magnetic properties of $\mathrm{Fe}_{3} \mathrm{O}_{4}$ and $\mathrm{Fe}_{3} \mathrm{O}_{4} /$ gold shell nanoparticles are characterized using SQUID magnetometer. Figure 3(b) shows the mass magnetization curve of $150 \mathrm{~nm} \mathrm{Fe} \mathrm{O}_{4}$ (blue line) and 150/25 nm Fe $3 \mathrm{O}_{4}$ core /gold shell (red line) nanoparticles versus the applied magnetic field at $300 \mathrm{~K}$ by cycling the field between -20 to $20 \mathrm{kOe}$. Both the magnetic core and magnetic core/gold shell nanoparticles are superparamagnetic at room temperature, showing no remanence or coercivity. The saturated mass magnetization of the core and core/shell nanoparticles at $300 \mathrm{~K}$ is 37.9 and $22.2 \mathrm{emu}^{-1}{ }^{-1}$, respectively. The saturated mass magnetization of magnetic core/gold shell nanoparticles are decreased due to the formation of gold shells.

As depicted in Figure 4, the dynamic and reversible as- (a)

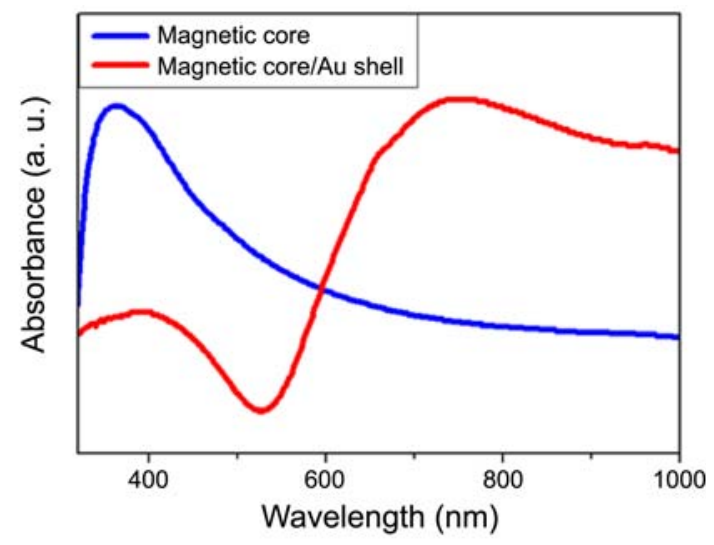

(b)

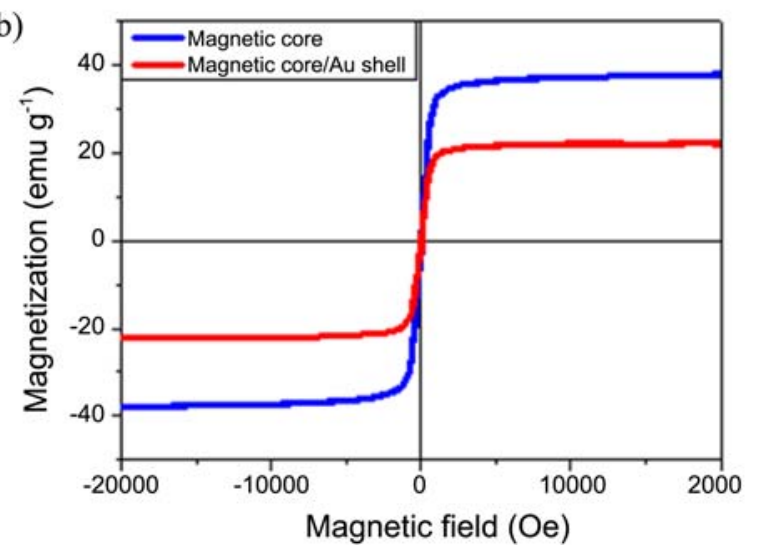

Figure 3. (a) The absorbance spectra of $\mathrm{Fe}_{3} \mathrm{O}_{4} /$ silica nanoparticle and gold shell-coated $\mathrm{Fe}_{3} \mathrm{O}_{4} /$ silica nanoparticle suspensions. The deposition of gold onto magnetic core leads to a shift in the SPR absorption. (b) The magnetization curves of magnetic core and magnetic core/gold shell particles. Both particles have superparamagnetic properties at room temperature.

sembly and arbitrary manipulation of gold 1D chains can be performed using the nanoparticles in an aqueous solution. (see Supporting movie) Without an applied magnetic field, the superparamagnetic core/gold shell nanoparticles are randomly dispersed in an aqueous solution. Under an external magnetic field, however, the nanoparticles are assembled to form 1D chain structures along the magnetic field lines to align the dipoles in the same direction. An attractive magnetic force due to the superparamagnetic core is balanced with repulsive electrostatic and solvation forces. Without a strong repulsion force, the particles irreversibly aggregate with one another when they are pushed together upon application of external magnetic fields. Since the magnetic core particles are aligned according to the direction of the applied magnetic field, we can easily manipulate the axis of gold chain structures simply by controlling the direction of the external magnetic field. Depending on the direction of the external magnetic field lines, the magnetic core/gold shell nanoparticles can be stood upright or laid out horizontally with respect to the surface of the glass substrate (Figure 4(b)). Also, after setting the magnetic field horizontally to align the particles parallel to the glass surface (xy plane), the external magnetic field can be rotated on the $z$ 
(a)
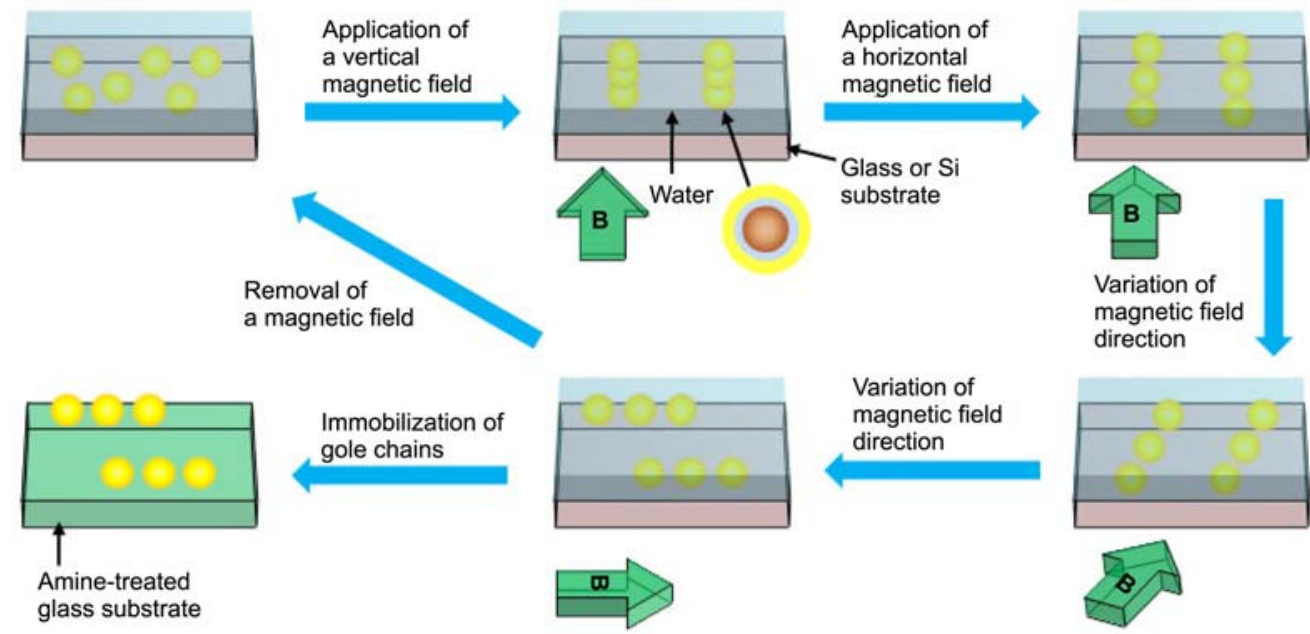

(b)
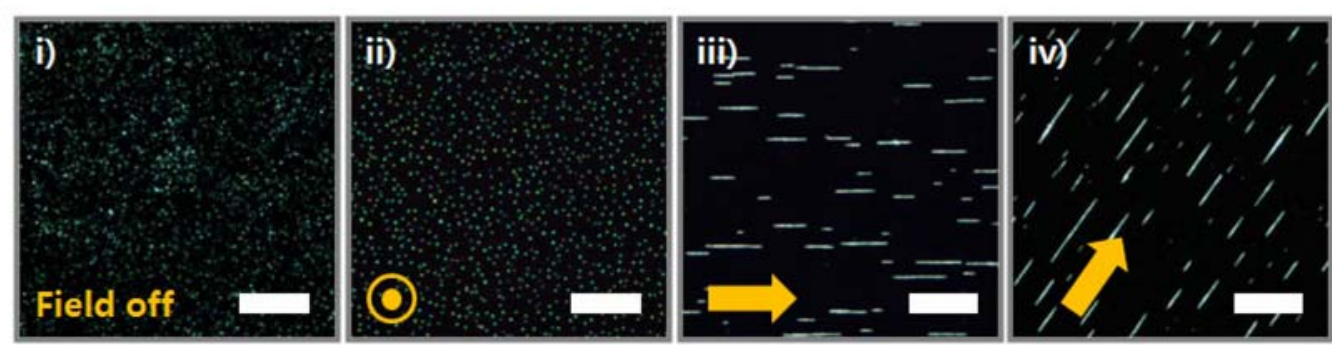

Figure 4. The formation and manipulation and immobilization of gold 1D chains. (a) A schematic diagram of dynamic and reversible formation and manipulation of gold 1D chains. (b) Reflection dark-field images of gold particle chain assemblies: (i) The particles are randomly dispersed in the absence of a magnetic field (ii-iv). When an external magnetic field is applied, gold nanoparticles are assembled to form chain structures along the magnetic field lines with a high degree of spatial control. The scale bars indicate $50 \mu \mathrm{m}$.

(a)

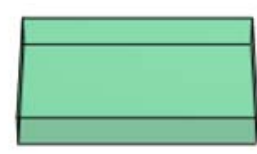

\section{APTES treatment \\ for introducing}

amine groups

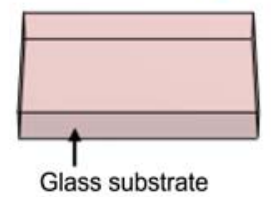

(b)

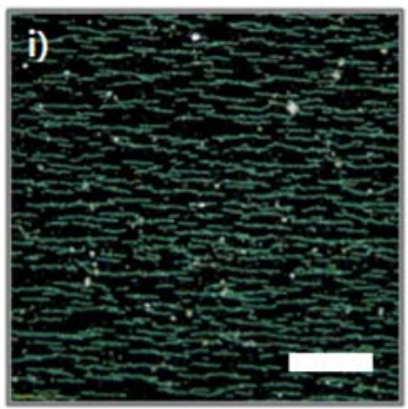

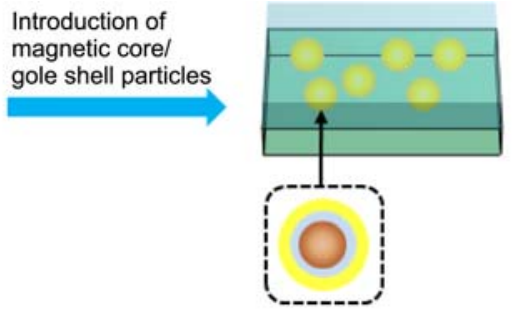

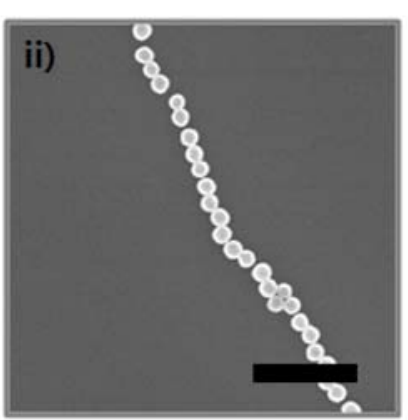

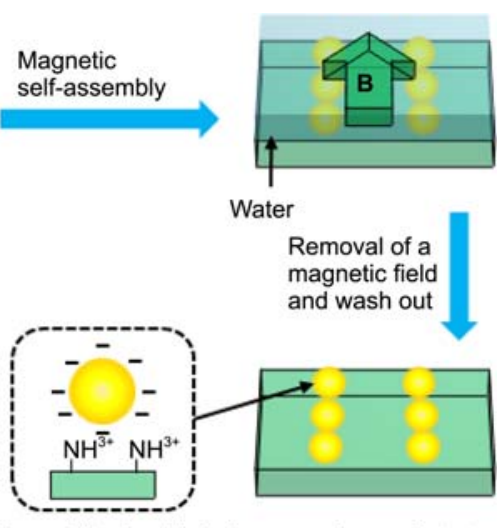

Immobilized gold chains on a glass substrate (c)

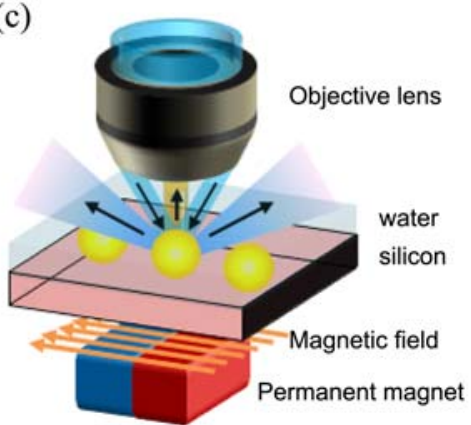

Figure 5. (a) Experimental procedures for immobilizing a gold 1D chain array on a glass substrate through combined use of a magnetic field and chemical assembly technique. (b) Reflection dark-field and FE-SEM images of immobilized $\mathrm{Fe}_{3} \mathrm{O}_{4}$ core/gold shell nanoparticle chains on a glass substrate. The scale bars indicate $50 \mu \mathrm{m}$ in Figure 5(b i) and $1 \mu \mathrm{m}$ Figure 5(b ii). (c) A schematic illustration of the reflection dark-field microscope setup. 
axis to rotate the direction of the chain axis. Therefore, compared with conventional methods for generating gold chain structures which utilize templates, this magnetic assembly approach enables simple and fast fabrication with a high degree of spatial control.

Once the properly oriented chain structures are obtained from magnetic self-assembly, the gold chains can be fixed on a glass substrate by the electrostatic interaction between the charged nanoparticle and amine groups of the substrate surface as depicted in Figure 5(a). ${ }^{32}$ The reflection dark-field and FE-SEM images of immobilized gold 1D chains on a glass substrate are shown in Figure 5(b). By functionalizing the glass surface with APTES, the well-defined 1D chain arrays are immobilized on the surface of the glass substrate without distortion. The gold shells are dominantly negatively charged due to the adsorption of anions and surfactant, while the amino groups are mainly positively charged. Therefore, the specific affinity originates from the strong electrostatic attractions.

\section{Conclusions}

In conclusion, we have developed magnetically controllable gold nanoparticles by synthesizing superparamagnetic $\mathrm{Fe}_{3} \mathrm{O}_{4}$ core/gold shell nanoparticles. The core/shell particles have the capability of forming gold $1 \mathrm{D}$ chains in the presence of an external magnetic field. Using this approach, millions of gold 1D chains can be assembled and oriented in along applied magnetic field lines. Furthermore, we provide a technique for immobilization of gold chain structures on a glass substrate. We believe that the magnetic self-assembly using the magnetic core/gold shell materials could provide possibilities for LSPR investigations such as a SERS substrate or for the construction of plasmon-based waveguides.

Acknowledgments. This work was partially supported by the National Research Foundation of Korea (NRF) grant funded by the Korea government (MEST) (No. 20120000045 and No. 2012-0009653). And also this work was partially supported by the IT R\&D program of MKE/KEIT [KI002126].

\section{References}

1. Sonnichsen, C.; Reinhard, B. M.; Liphardt, J.; Alivisatos, A. P. Nat. Biotechnol. 2005, 23, 741.

2. Prodan, E.; Radloff, C.; Halas, N. J.; Nordlander, P. Science 2003, $302,419$.
3. Shi, W. L.; Sahoo, Y.; Swihart, M. T.; Prasad, P. N. Langmuir $\mathbf{2 0 0 5}, 21,1610$

4. Liu, G. L.; Yin, Y. D.; Kunchakarra, S.; Mukherjee, B.; Gerion, D.; Jett, S. D.; Bear, D. G.; Gray, J. W.; Alivisatos, A. P.; Lee, L. P.; Chen, F. Q. F. Nat. Nanotechnol. 2006, 1, 47.

5. Reinhard, B. M.; Sheikholeslami, S.; Mastroianni, A.; Alivisatos, A. P.; Liphardt, J. P Natl. Acad Sci. USA 2007, 104, 2667.

6. Hayward, R. C.; Saville, D. A.; Aksay, I. A. Nature 2000, 404, 56.

7. Talley, C. E.; Jackson, J. B.; Oubre, C.; Grady, N. K.; Hollars, C. W.; Lane, S. M.; Huser, T. R.; Nordlander, P.; Halas, N. J. Nano Lett. 2005, 5, 1569.

8. Gu, G. H.; Suh, J. S. J. Phys. Chem. C 2010, 114, 7258.

9. Tabor, C.; Murali, R.; Mahmoud, M.; El-Sayed, M. A. J. Phys. Chem. A 2009, 113, 1946.

10. Kim, S.; Shuford, K. L.; Bok, H. M.; Kim, S. K.; Park, S. Nano Lett. 2008, 8, 800 .

11. Maier, S. A.; Kik, P. G.; Atwater, H. A.; Meltzer, S.; Harel, E.; Koel, B. E.; Requicha, A. A. G. Nat. Mater. 2003, 2, 229.

12. Hu, M. S.; Chen, H. L.; Shen, C. H.; Hong, L. S.; Huang, B. R.; Chen, K. H.; Chen, L. C. Nat. Mater. 2006, 5, 102.

13. Simon, U. Adv. Mater. 1998, 10, 1487.

14. Burkett, S. L.; Mann, S. Chem. Commun. 1996, 321.

15. Meister, A.; Drescher, S.; Mey, I.; Wahab, M.; Graf, G.; Garamus, V. M.; Hause, G.; Mogel, H. J.; Janshoff, A.; Dobner, B.; Blume, A. J. Phys. Chem. B 2008, 112, 4506.

16. Sardar, R.; Shumaker-Parry, J. S. Nano Lett. 2008, 8, 731.

17. Warner, M. G.; Hutchison, J. E. Nat. Mater. 2003, 2, 272.

18. Gao, X. Y.; Djalali, R.; Haboosheh, A.; Samson, J.; Nuraje, N.; Matsui, H. Adv. Mater. 2005, 17, 1753.

19. Ellis, A. V.; Vjayamohanan, K.; Goswaimi, R.; Chakrapani, N.; Ramanathan, L. S.; Ajayan, P. M.; Ramanath, G. Nano Lett. 2003, 3,279 .

20. Lalatonne, Y.; Richardi, J.; Pileni, M. P. Nat. Mater. 2004, 3, 121.

21. Hermanson, K. D.; Lumsdon, S. O.; Williams, J. P.; Kaler, E. W.; Velev, O. D. Science 2001, 294, 1082.

22. Vernhes, M. C.; Cabanes, P. A.; Teissie, J. Bioelectroch. Bioener. 1999, 48, 17.

23. Spasova, M.; Salgueirino-Maceira, V.; Schlachter, A.; Hilgendorff, M.; Giersig, M.; Liz-Marzan, L. M.; Farle, M. J. Mater. Chem. $\mathbf{2 0 0 5}, 15,2095$.

24. Kim, H.; Ge, J.; Kim, J.; Choi, S.; Lee, H.; Lee, H.; Park, W.; Yin, Y.; Kwon, S. Nat. Photonics 2009, 3, 534.

25. Lee, H.; Kim, J.; Kim, H.; Kim, J.; Kwon, S. Nat. Materials 2010, 9,745 .

26. Ge, J. P.; Lee, H.; He, L.; Kim, J.; Lu, Z. D.; Kim, H.; Goebl, J.; Kwon, S.; Yin, Y. D. J. Am. Chem. Soc. 2009, 131, 15687.

27. Ge, J. P.; He, L.; Goebl, J.; Yin, Y. D. J. Am. Chem. Soc. 2009, 131,3484

28. Ge, J. P.; Yin, Y. D. Adv. Mater. 2008, 20, 3485.

29. Brinson, B. E.; Lassiter, J. B.; Levin, C. S.; Bardhan, R.; Mirin, N.; Halas, N. J. Langmuir 2008, 24, 14166.

30. Yong, K. T.; Sahoo, Y.; Swihart, M. T.; Prasad, P. N. Colloid Surface A 2006, 290, 89.

31. Lim, J.; Eggeman, A.; Lanni, F.; Tilton, R. D.; Majetich, S. A. $A d v$. Mater. 2008, 20, 1721.

32. Zheng, J. W.; Zhu, Z. H.; Chen, H. F.; Liu, Z. F. Langmuir 2000, 16,4409 . 